\title{
Carebit: A Mobile App for Remote Informal Caregiving
}

\section{Zachary Shea}

University of Central Florida

Orlando, FL

zshea@knights.ucf.edu

\section{David Taylor}

University of Central Florida

Orlando, FL

david.taylor@knights.ucf.edu

\author{
Zaina Aljallad \\ University of Central Florida \\ Orlando, FL \\ zaina.aljallad@knights.ucf.edu
}

\section{Chhaya Chouhan}

University of Central Florida

Orlando, FL

chhayachouhan@knights.ucf.edu

Pamela J. Wisnewski

University of Central Florida

Orlando, FL

pamwis@ucf.edu

\section{ABSTRACT}

Assistive technologies have been developed to make informal caregiving more feasible, but are typically privacy invasive, unaffordable, or inconvenient. To address this gap, we present Carebit, a cross-platform app for iOS and Android users that allows caregivers to be notified based on preset thresholds about their loved one (caregivee). We have designed Carebit to protect the privacy of the caregivee while allowing the caregiver to conveniently check up on them through their phone. Carebit also provides an app for the caregivee to be able to communicate with their caregiver.

Permission to make digital or hard copies of part or all of this work for personal or classroom use is granted without fee provided that copies are not made or distributed for profit or commercial advantage and that copies bear this notice and the full citation on the first page. Copyrights for third-party components of this work must be honored. For all other uses, contact the Owner/Author.

CSCW '19 Companion, November 9-13, 2019, Austin, TX, USA

(C) 2019 Copyright is held by the owner/author(s).

ACM ISBN 978-1-4503-6692-2/19/11.

https://doi.org/10.1145/3311957.3359511 


\section{ACM CLASSIFICATION}

\section{KEYWORDS}

- Human-centered computing Usability testing

\section{KEYWORDS}

Assistive technology; Caregiving;

Mobile; Privacy.

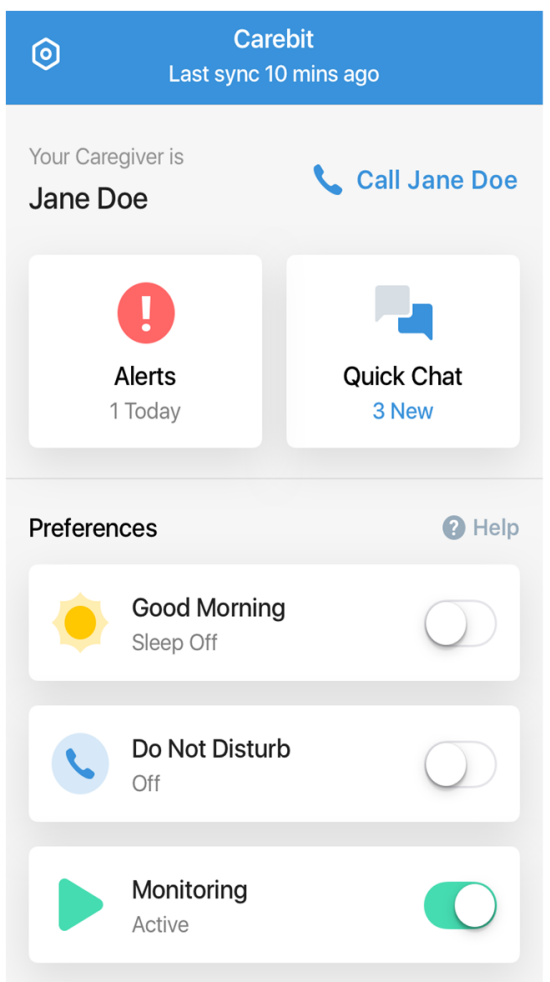

Figure 1: Carebit Dashboard (Caregivee)

\section{INTRODUCTION}

Over 43 million people in the United States spend, on average, about 24 hours each week providing informal care for their loved one. An informal caregiver is a family member that provides services and supervision to a loved one, who may be ill or otherwise need help [1]. Informal caregivers often take care of loved ones as in-home patients, requiring constant attention that creates significant caregiver burden, especially when care requires constant supervision [2]. As a result, a number of tele-monitoring systems to help with in-home care have been developed [3]. Yet, a major concern with these monitoring systems is that they often require patients to give up their personal privacy for the sake of their health and safety [4]. To help support caregivers and their caregivees, we conceptualized and created Carebit, a mobile application ("app") which leverages the Fitbit API [5], for informal caregiving relationships, where the patient is well enough to live independently but desires a lightweight means for another to check in on them on a daily basis [6]

\section{RELATED WORK}

Studies have been conducted to understand how much caregivees really care about their privacy. Talebi et. al. conducted a study to understand how patients view their privacy [7] and found that most patients desired some type of privacy customization along with the ability to control who has access to their personal information.

In another study, participants were presented with a system called SHEI (Safe Home Living). This system was designed to be used in "less than emergency" situations where patients didn't have a life-threatening cause, so their caregivers were able to come and provide their help. Many patients were concerned with their privacy, not wanting their children or loved one to know what they are doing every moment of the day. In the study, patients also admitted to feeling embarrassed and losing a sense of independence by having someone watch them [4]. Choe et. al. presented scenarios where it would be useful for patients to be monitored by their doctors to either keep them living a healthier life or helping them diagnose symptoms. However, monitoring someone can affect their personal interactions, workflow, and communications dynamic [8]. There were positive and negative results to these scenarios, however they still present the benefits to having someone to be able to monitor progress.

While other technologies have been developed, they are still prone to invade caregivee's privacy. Therefore, we wanted to create a balance between privacy vs healthcare through Carebit. Carebit focuses on putting the caregivee's privacy and comfort first. We accomplish this task by having a separate app for caregivees, where they can choose when, who, and what they want to share their information with. Caregivees are able to turn off sharing at any time. We present this contribution to $\mathrm{CSCW}$ as a novel idea to help caregivers and their caregivees alike. We hope to continue the 


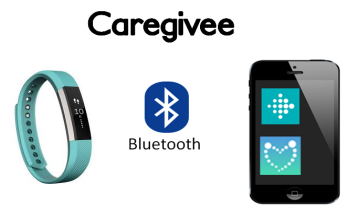

Fitbit syncs to Fitbit app through Bluetooth to smartphone

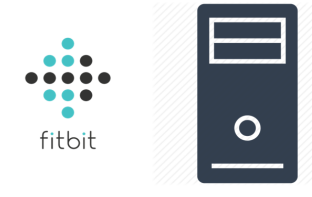

Fitbit app syncs to Fitbit server through wi-fi/data
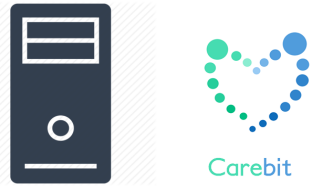

Fitbit server syncs to Carebit server through service

\section{Caregiver}

Carebit app syncs with server to send alerts to caregiver

Caregiver communicates with caregivee via Carebit app

Figure 2: System Diagram conversation around informal caregiving and how to have low cost and non-intrusive technologies help monitor caregivees.

Below we discuss the design and implementation of Carebit

Carebit provides an interface for both the caregiver and caregivee, it works with the caregivee's Fitbit to simplify caregiving on both sides. Carebit provides an interface for caregivers to monitor vital health functions of the caregivee using their Fitbit. The caregiver is also able to choose when they would like to receive notifications or health alerts about their caregivee. By leveraging the Fitbit API, Carebit is able to synchronize with Fitbit and provide near real-time push notifications to the caregivers based on customizable alert thresholds for health data. Carebit also provides a dashboard for the caregivee as seen in Figure 1, which allows for a more social experience with the caregiver. It provides features for messages and quick interactions, "do not disturb" settings, control of the data being collected, verification of alerts (e.g. when a high heart rate isn't actually a critical situation), and emergency notifications.

\section{CAREBIT DESIGN}

The caregiver's application focuses on displaying the caregivee's health data and receiving push notifications in the event of a critical situation (determined by the user's settings). When first configuring a caregivee, we offer three preset health profile options: 1) Active 2) Sedentary and 3) Homebound. These profiles provide defaults for heart rate and step thresholds, as well as notification levels. We also allow the caregiver to customize these thresholds, should they choose to do so. Caregivers will then be taken to a dashboard (Figure 3) where they are able to view the caregivee's vitals. These include heart rate and footsteps. Alerts are also presented on this screen; caregivers are able to contact their loved one directly if needed.

Currently there is no way to tell when someone is sleeping in real-time through Fitbit without a further heart-rate analysis and algorithm. To combat this, we have given caregivees the ability to let their caregivers know when they are going to sleep.

\section{CAREBIT IMPLEMENTATION}

The foundation of Carebit is the API and database that receives the caregivee's data from Fitbit and presents it to the caregiver. The Carebit API is a REST (REpresnetational State Transfer) API written in Python with the Flask framework, and the SOLAlchemy library to interact with a MySQL database. The API and database are hosted on an Ubuntu Linux 18.10 VPS (Virtual Private Server) provided by DigitalOcean. Communication between the client applications and the API is accomplished with HTTP requests and JSON (JavaScript Object Notation) objects. A visualization of this is shown in Figure 2. 


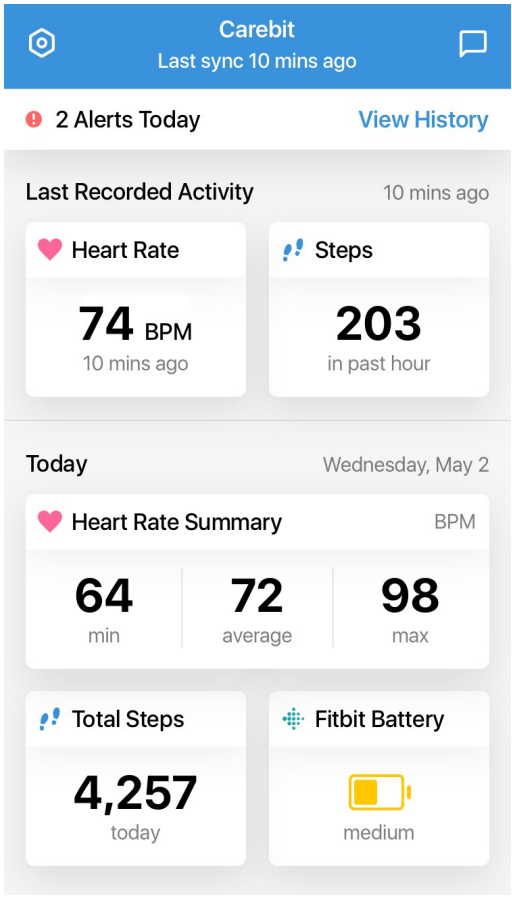

Figure 3: Carebit Dashboard

(Caregiver)

\section{Alert Notifications}

The following notifications can be customized from the caregiver's phone:

- High Heart Rate

- Low Heart Rate

- No Heart Rate

- Too Many/Few Steps

- No Steps

- Low Fitbit Battery

- $\quad$ Time Without Sync
The API also holds the caregivee's privacy settings, health data thresholds, and when to send push notifications.

- Push Notifications - the Carebit API makes use of Google Firebase. This allows for an easy to use interface for both iOS and Android notifications. Push notifications are a major feature in Carebit, as it notifies caregivers of critical situations.

When a caregivee's data is received, the readings are compared to the thresholds set by the caregiver, and if those thresholds are exceeded, then a notification is sent to the caregiver. The types of alerts that can be received are listed on the side bar.

- Thresholds - Carebit provides thresholds for low heart rate, high heart rate, time without heart rate, too many steps, time without steps, and Fitbit low battery.

- Synchronization - the caregivee's Fitbit app will synchronize with the Fitbit backend as often as every 15 minutes, as long as the Fitbit is in range of the caregivee's phone and there is adequate $\mathrm{Wi}-\mathrm{Fi}$ or mobile data connectivity. However, it could sync more often, if the caregivee opens the Fitbit app.

When the sync happens, the Fitbit Subscriptions API will then send an HTTP POST request to our configured API endpoint. The POST request to our configured API endpoint. The POST request will contain the Fitbit user IDs of any configured subscriber who has available data, as well as which data is available (e.g. heart rate, steps, etc.).

\section{CONCLUSION}

We believe Carebit represents a shift from invasive monitoring technologies to a more collaborative caregiving relationship. By keeping the caregivee involved in every part of the caregiving process, the are always apart of the process. From an aging loved one, to a child on the Autism Spectrum, Carebit can benefit a wide range of caregiving relationships and provide a welcome peace of mind in an often-stressful situation.

\section{ACKNOWLEDGEMENTS}

The authors wish to acknowledge the contributions of Rangita Rajakumar, Kaley Brindisi, Kim Chen, Ben Faria, Ian Harvey, and Guensly Louis, who developed the original Carebit application, the foundation upon which our version of Carebit is based. Support for this work was provided by the National Science Foundation Research Experience for Undergraduates program under Award No. 1560302 and The Learning Institute for Elders at University of Central Florida, Inc. Any opinions, findings, and conclusions and recommendations expressed in this material are those of the author(s) and do not necessarily reflect the views of the National Science Foundation. 


\section{REFERENCES}

[1] "Caregiver Statistics: Demographics | Family Caregiver Alliance." [Online]. Available: https://www.caregiver.org/caregiver-statistics-demographics. [Accessed: 12-Jun-2019].

[2] A. Williams et al., "A Canadian qualitative study exploring the diversity of the experience of family caregivers of older adults with multiple chronic conditions using a social location perspective," Int. J. Equity Health, vol. 15, p. 40, Mar. 2016.

[3] C. Ní Scanaill, S. Carew, P. Barralon, N. Noury, D. Lyons, and G. M. Lyons, "A review of approaches to mobility telemonitoring of the elderly in their living environment," Ann. Biomed. Eng., vol. 34, no. 4, pp. 547-563, Apr. 2006.

[4] J. Vines et al., "Making Family Care Work: Dependence, Privacy and Remote Home Monitoring Telecare Systems," in Proceedings of the 2013 ACM International Joint Conference on Pervasive and Ubiquitous Computing, New York, NY, USA, 2013, pp. 607-616.

[5] "Fitbit Development: Fitbit SDK." [Online]. Available: https://dev.fitbit.com/. [Accessed: 17Jun-2019].

[6] A. K. Ghosh, Z. Aljallad, K. Badillo-Urquiola, and P. Wisniewski, "Carebit: A PrivacyPreserving 'Step' Toward Remote Informal Caregiving," in Proceedings of the 2018 ACM Conference on Supporting Groupwork, New York, NY, USA, 2018, pp. 154-157.

[7] N. Talebi, C. Hallam, and G. Zanella, "The new wave of privacy concerns in the wearable devices era," in 2016 Portland International Conference on Management of Engineering and Technology (PICMET), 2016, pp. 3208-3214.

[8] E. K. Choe, B. Lee, T. O. Andersen, L. Wilcox, and G. Fitzpatrick, "Harnessing the Power of Patient-Generated Data,” IEEE Pervasive Comput., vol. 17, no. 2, pp. 50-56, Apr. 2018. 